Mark Toraason · Charles Hayden · Dave Marlow

Richard Rinehart · Patty Mathias · Dwight Werren

D. Gayle DeBord · Thomas M. Reid

\title{
DNA strand breaks, oxidative damage, and 1-0H pyrene in roofers with coal-tar pitch dust and/or asphalt fume exposure
}

Published online: 13 February 2002

(c) Springer-Verlag 2002

\section{Int Arch Occup Environ Health (2001) 74:396-404}

Due to an unintentional oversight three authors' names (Larry D. Olsen, Charles E. Neumeister, and Elaine S. Mathews) were left off the paper; furthermore, due to an unfortunate misunderstanding of their contributions two others (Kenneth L. Cheever and Kate L. Marlow), who were merely acknowledged, should have been credited as authors. The correct listing of authors is thus as follows:

Mark Toraason, Charles Hayden, Dave Marlow, Richard Rinehart, Patty Mathias, Dwight Werren, Larry D. Olsen, Charles E. Neumeister, Elaine S. Mathews, Kenneth L. Cheever, Kate L. Marlow, D. Gayle DeBord, Thomas M. Reid.

In the address section, the authors Olsen, Neumeister, Mathews, Cheever, and K. Marlow should be included as follows:

M. Toraason $(\bowtie)$, C. Hayden, D. Marlow, P. Mathias, D. Werren, D.G. DeBord, T.M. Reid, L.D. Olsen, C.E. Neumeister, E.S. Mathews, K.L. Cheever, K. L. Marlow.

National Institute for Occupational Safety and Health, 4676 Columbia Parkway, Cincinnati, OH 45226, USA.

On page 398, the paragraph starting at the bottom of the first column should be replaced with:

Twenty-three of the roofers agreed to wear personal air monitors that collected samples from their breathing zone. Personal breathing zone samples were analyzed for total particulates and the benzene-soluble fraction by DataChem Laboratories, Salt Lake City, Utah, USA, using NIOSH Method 5042 (NIOSH 1998). PACs were measured by a modified version of NIOSH Method 5800 (NIOSH 1998). Low-molecularweight PACs (2- and 3-ring compounds) were measured at spectrofluorometer settings of 254-nm excitation and 360-nm emission (see footnote). Highmolecular-weight PACs (4- and 5-ring compounds including pyrene) were measured at spectrofluorometer settings of 254-nm excitation and 400-nm emission (see footnote). Workers wore the air monitors during the entire work shift. The time-weighted average (TWA) concentration $\left(\mathrm{mg} / \mathrm{m}^{3}\right)$ was calculated for each day of the entire air-monitoring period, and the daily values were used to calculate an average weekly TWA for each roofer.

On page 398, at the end of the second column, a footnote should be inserted that reads as follows:

While the emission wavelengths are selective for either 2- and 3-ring PACs or 4- and 5-ring PACs, the measurements are not mutually exclusive because both classes of PACs contribute to the signal.

In the acknowledgements, the phrase "Ken Cheever and Kate Marlow for analytical assistance" should be deleted.

In the references, the journal title for Hatjian et al. 1997 should be changed to J Occup Health Saf Aust NZ.

In the references, the reference listing for NIOSH (1994) should be replaced with NIOSH (1998) NIOSH Manual of Analytical Methods (NMAM), 4th edn, 2nd Supplement. Cincinnati, Ohio: National Institute for Occupational Safety and Health, DHHS (NIOSH) 98-119.

M. Toraason $(\varangle) \cdot$ C. Hayden $\cdot$ D. Marlow $\cdot$ P. Mathias

D. Werren · D.G. DeBord · T.M. Reid

National Institute for Occupational Safety and Health,

4676 Columbia Parkway, Cincinnati, OH 45226, USA

R. Rinehart

Harvard School of Public Health,

Boston, Massachusetts, USA 